\title{
Industry and Policy: Partnerships in Disruptive Times
}

\section{Leendert van Bochoven}

IBM Industry Academy, http://www-935.ibm.com/industries/industryacademy/

\begin{abstract}
The rate of (technological) change in today's dynamic environment calls for new policies and collaboration models between governments and industry. Two key elements will underpin successful policies for dealing with innovation and the impact of technology: an innovation ecosystem and an innovation platform. Just like companies are involving customers in private sector innovation, governments are seeking to involve citizens. There is a growing trend to engage citizens more and more in the co-creation of public services. The citizen co-creation approach also has merits for the defense and security industry, and there are several successful examples showcasing new ways of collaboration, overcoming the traditional obstacles.

Three key recommendations will enable governments to overcome innovation challenges. These recommendations depend on two essential enablers to deal with disruptive innovation in government organizations: an innovation ecosystem and an innovation platform. Without both, innovation is for sure going to fail. Given the rate of unprecedented technological change, governments, militaries and businesses have to find creative ways to work and innovate together.
\end{abstract}

Keywords: Policy, industry, technology, innovation, ecosystem, cognitive, public-private collaboration, partnerships, co-creation.

The world is going through a number of unprecedented changes, including in geopolitics, technology, and the climate. This dynamic environment calls for new forms of collaboration between government and industry, as the traditional arm's-length client-provider relationship is not responsive enough for today's rapid pace of change. The existing collaboration models are running 
out of steam. Where in the past government collaboration was limited to small coalitions of like-minded partners, for today and tomorrow there must be a change in collaboration models beyond the typical approach. These new collaboration models require different policies to be workable in the government space. This article will explore key elements of these policies. Most are not new and have been called for many times, but the urgency to implement them keeps increasing due to the changes in the world. Two key elements will underpin successful policies for dealing with innovation and the impact of technology: an innovation ecosystem and an innovation platform.

\section{Making the Case for Change}

Many reports describe an emerging picture of a mind-boggling number of devices and sensors connected to the Internet. For example, Gartner predicts that by 2020, 35 billion objects will be online. Already in 2016, the spending on new Internet of Things (IoT) hardware will exceed \$2.5 million per minute. ${ }^{1}$ The digital and physical world will continue to integrate and become increasingly interconnected. Physical things will have a digital layer around them, and each of these things will have a digital footprint and thus generate an incredible volume of data. Not only will the volume of data increase, but the nature of the data will change as well. This is disrupting existing approaches to computing while opening vast new opportunities to create value.

That is especially true of "edge data," which includes all the new forms of data generated by users and their devices, such as tablets, smartphones, sensors and more. It is fast-paced, dynamic, unstructured, temporal in nature, unlike any prior data creation model. Edge data is incredibly rich in offering an understanding of context and, therefore, has potentially very high value. This is but one example of a technology trend that already has a big impact on organizations, and there is rather a confluence of developments. Each of the trends in nano, bio and information technology will have its own line of development. Information technology alone will have a disruptive effect, and certainly when it is combined with new possibilities in nano and bio technology.

\section{Partnership Models in the Age of Disruptive Innovation}

Management guru Clayton Christensen coined the term "disruptive technologies" in his book, "The Innovators Dilemma," in 1997. ${ }^{2}$ This was later followed by the term "disruptive innovation" to describe how new entrants target the bottom of a market and then relentlessly move up market, eventually ousting established providers. However, what was once a relatively rare phenomenon

1 Gartner, "Forecast: Internet of Things, Endpoints and Associated Services, Worldwide," 29 October 2015, available at https://www.gartner.com/doc/3159717/ forecast-internet-things--endpoints.

2 Clayton Christensen, The Innovators Dilemma: When New Technologies Cause Great Firms to Fail (Boston, MA: Harvard Business Review Press, 1997). 
has now become a regular occurrence. Innovations that harness new technologies or business models, or exploit old technologies in new ways, are emerging on an almost daily basis. Disruptive is not a popular word in government circles, but the accelerating digitization and impact of radical technology changes are certainly also disrupting government.

In 2012, IBM's Institute for Business Value (IBV) conducted its fifth biennial "Global CEO Study." ${ }^{3}$ This was based on more than 1700 interviews with CEOs from 64 countries across 18 industries, including government. As part of the analysis, the IBV sought to understand differences between responses of CEOs in outperforming organizations and those in underperforming organizations. According to the study, of all the external forces that could impact their organizations over the next three to five years, CEOs see change in technology as the most critical, as technological factors are by far the biggest of the various external forces buffeting their organizations. Technology was at the top of the list back in 2012, and the view is no different in the 2015 CEO study. ${ }^{4}$ For governments, the impact of budgets topped the list ( $89 \%$ of the government leaders cited this as the most important factor) and technological factors (78\%) as the second external factor influencing government organizations.

Meanwhile, the 2015 CEO study, "Redefining Boundaries," examines how businesses are responding to these new disruptive innovations. A few years ago, business leaders could see the competition coming. The biggest risk was the advent of a new rival with a better or cheaper product or service. The threat could be offset by improving or expanding the range of products and services on offer, or getting to market more efficiently and imaginatively. Nowadays companies ask themselves if they are about to be "Ubered." While most government organizations are not faced with these competitive challenges, there are both implications for governments and lessons to be learned from how market leaders are coping with these innovation challenges. There are two major implications and takeaways for government leaders. Firstly, the strategies and tactics that will enable private sector organizations to effectively compete amidst the disruption of industry convergence can also enable government organizations to become more agile, effective and efficient while also improving innovation capacity. Government organizations can draw on lessons from the private sector to help transform business and operating models. Secondly, governments and key actors in the public sector (e.g. educational institutions, economic development and investment promotion organizations) must create business environments that enable private sector companies to thrive

3 IBM Institute for Business Value, Leading Through Connections: Insights from the Global Chief Executive Officer Study (IBM, 2012), available at http://www935.ibm.com/services/multimedia/anz_ceo_study_2012.pdf.

4 IBM Institute for Business Value, Redefining Boundaries: The Global C-suite Study (IBM, 2015), available at www-935.ibm.com/services/c-suite/study/pdf/ibm_global_ csuite_study-2015.pdf. 
amidst this disruption to ensure economic vitality and sustainable economic growth in regional economies.

While both takeaways apply to government in general, they equally apply to the defense and security sector. Defense and intelligence organizations must collaborate much more closely with industry in order to tackle disruptive technologies and innovate the "business of security," especially because their adversaries also have access to most of these technologies, without the burden of lengthy acquisition processes. State and non-state actors apply and exploit innovative technologies in order to disrupt the security environment and challenge the status quo. For example, just a few years ago cyber security concerns were just blips on the radar screen. Today, the majority of business, government and military leaders, irrespective of role or the technology they selected, think cyber security is a top risk.

\section{Amplifying Innovation with Partnerships}

The changing landscape has led to the question of what government organizations are doing to deal with external forces and how they ensure they outperform their peers. The 2012 IBV study concluded that one the three imperatives essential for outperformance is "amplifying innovation with partnerships." 5 This was further reflected in the survey, as nearly 70 percent of CEOs responded that they are aiming to pursue extensive partnerships.

Rising complexity and escalating competition have made partnering a core innovation strategy for many organizations, but to enable sustained, fruitful innovation partnerships, organizations will need deeper, more integrated relationships. Partner organizations will have to share collaborative environments, data and control. They will need to enable close working relationships among staff, and not just executives. Even when an organization is performing well, CEOs must occasionally break from the status quo and introduce new external catalysts, unexpected partners and some intentionally disruptive thinking. The same holds true for government organizations.

The aforementioned study reveals three new ways in which organizations can connect with partners to accelerate innovation. ${ }^{6}$ The first is to fundamentally change how to partner. As the pressure to innovate mounts, organizations are reevaluating how they engage partners. This is also necessary because of the increasing costs to innovate. These costs are not always visible in an organization, but certainly have to be taken into account when discussing new approaches to partnering. They are often also overlooked in partnerships between the government and private sector and can become a real stumbling block for sustainable partnering models.

Partnership models can achieve differentiation through social innovation by extending communication and collaboration tools. Peers can interact within

5 IBM Institute for Business Value, Leading Through Connections, page 43 onwards.

6 Ibid., 48-50 
and across organizations, allowing for integration of data resources to reveal unexpected, mutually beneficial insights. The boundaries between organizations are becoming more porous, while interactions span more functions and are more continuous. A good example in the NATO context is the Innovation Hub of NATO's Allied Command Transformation, which enables collaboration among a wide range of partners. Another way to change the partnership model is to expand the scope of the partnerships. Organizations should evaluate ways to extend and connect existing partnerships on innovation to include ideation, research and development and sales, marketing or human resources. The partnership model must also address the governance challenge. This may prove to be the most difficult to tackle, especially in partnership models between the government and private sector. The partnership model should establish ways to share key aspects of control, such as prioritization, decision-making and funding, that are traditionally dominated by one partner. As the costs to innovate increase, the need for transparency is also rising. Control and governance must increasingly be shared.

A second way organizations can connect with partners to accelerate innovation is to make partnerships personal. ${ }^{7}$ Technology now presents opportunities for much deeper connections with partners, while this interconnectedness allows for more opportunities for innovation - both spontaneous and orchestrated. To this end, the responsibility for managing partnerships can be broadened within the organization, as it is not just the responsibility of a single unit in the organization. This means that the capability for relationship management must be embedded across the organization and use centralized alliance management functions to supply specialized skills. In US military terms: partnerships are not just the responsibility of the senior officer in charge of Civil-Military Co-Operation and Interagency Partnering, but should be implemented across the entire joint command structure. Further, fostering relationships at each level across partnering organizations provides avenues to develop personal connections among peers. These partners could be a community of people rather than organizations; the view should not be limited to organizations, as the most valuable partnership might be with a group of individuals.

Thirdly, another way organizations can work with partners to accelerate innovation is by breaking collaboration boundaries. To address rising societal and technological complexity, organizations need to look beyond traditional partners and conventional views on innovation for new inspiration and necessary capabilities. Organizations should explore unconventional partnerships and study nontraditional alliances emerging in other industries. There may be parallels with some industries that can integrate capabilities not commonly found in others. In a similar vein, one of the most difficult challenges is to think like a disruptor, as existing structures and governance models make it difficult to think beyond business as usual, especially when business as usual is working.

7 Ibid., 49. 
Disruptors question the norms and introduce new stimulation from the outside. Finally, it is beneficial to approach untenable issues or grand challenges by partnering across the entire system, namely with governments, non-governmental organizations or even with competitors.

\section{Partnership Models for the Co-creation of Public Services}

Just as companies are involving customers in private sector innovation, governments are seeking to involve citizens. There is a growing trend to engage citizens in the co-creation of public services. Three broad issues have made it imperative for government agencies to change their relationship with citizens in problem-solving:

- Ongoing budgetary pressure motivates new, less resource intensive modes of problem-solving in government.

- The complex nature of the problems calls out for more collaborative approaches that involve external partners, including citizens.

- New technologies make connecting with citizens easier and reduce the cost of such collaboration in problem solving. ${ }^{8}$

The 2013 Study by the IBM Center for the Business of Government conducted by Satish Nambisan and Priya Nambisan outlines four distinct roles citizens can play in public service co-creation and problem-solving: explorers, ideators, designers and diffusers. As explorers, citizens can identify, discover and define emerging and existing problems in public services. ${ }^{9}$ A good example of this is the e-People initiative in South Korea, which allows citizens to voice their concerns and ideas through e-petitions. The objective is to "make a new face of Korea by resolving even trivial complaints after listening closely to the voices of the people and accepting their creative ideas positively." 10

As ideators, citizens can conceptualize novel solutions to well-defined problems in public service. Challenge.gov is an example of this. The initiative provides a listing of challenge and prize competitions, all of which are run by more than 80 agencies across the US federal government. As stated on the Challenge.gov website: "These include technical, scientific, ideation, and creative competitions where the US government seeks innovative solutions from the public, bringing the best ideas and talent together to solve mission-centric problems." Challenge.gov has offered more than $\$ 220$ million in prize money since 2010.

Next, as designers, citizens are able to design and/or develop implementable solutions to well-defined problems in public service. For example, citizens

8 Satish Nambisan and Priya Nambisan, Engaging Citizens in Co-Creation in Public Services: Lessons Learned and Best Practices (IBM Center for the Business of Government, 2013).

9 Ibid.

${ }^{10}$ https://www.epeople.go.kr/jsp/user/on/eng/intro01.jsp. 
may develop applications, or apps, based on open government data, as governments are adopting open data strategies to enable citizens to build innovative solutions. For example, data.gov.uk shows an extensive list of apps built on open data. The top rated app, Fasteroute, provides users with real time information about train departures and arrivals on the national rail network. The Route Risk app is another very useful app that analyses the safety of roads based on road safety data from the UK Department for Transport.

Finally, as diffusers, citizens may support or facilitate the adoption and diffusion of public service innovations among specific target populations. This is very similar to launching customers in the private sector.

The four roles imply different types of government vs. citizen interaction and thus require different approaches and mechanisms to support them. These approaches and mechanisms depend on the innovation ecosystem and the innovation platform.

\section{Innovation Partnership Models in Defense and Security}

The citizen co-creation approach also has merits for the defense and security industry, although the sensitive nature of the problems and solutions limit certain government interactions. There are several examples of comparable strategies in the defense and security sector. One major example is the Network and Information Sciences International Technology Alliance (ITA) - a collaborative research alliance between the UK Ministry of Defence (MOD), US Army Research Laboratory (ARL) and a consortium of more than 20 leading academic and industry partners. ${ }^{11}$ The ITA program started in 2006 with the strategic goal of producing fundamental advances in information and network sciences that will enhance decision-making for coalition operations, enable rapid, secure formation of ad hoc teams in coalition environments and enhance US and UK capabilities to conduct coalition warfare. The first phase of the ITA program concluded in 2011, and now the program is in its second phase. The ITA brings an extensive number of players together and focuses on specific defense issues. The outcomes of the research are available to all participating organizations and several findings made their way into the public domain in the form of an extensive list of published papers, so that organizations can take these findings forward into new products and solutions.

Meanwhile, the US Department of Defense (DoD) Defense Advanced Research Projects Agency has begun using InnoCentive as a platform for innovation. ${ }^{12}$ InnoCentive@Work is collaborative innovation management software that enables organizations to engage diverse innovation communities such as employees, partners or customers to help rapidly generate novel ideas and solve the most pressing problems. Commercial organizations and government agencies use the platform to crowdsource challenges through collaboration

11 http://www.usukita.org.

12 http://www.innocentive.com. 
with individuals, communities and networks. The DoD has also reached out to innovation from different sources opening offices in Silicon Valley in a project called DIUx. In the competitive and fast-moving technological environment, the DoD hopes "DIUx will help to cultivate and facilitate a lasting relationship with new innovators, initially in Silicon Valley, and those who don't always work with DoD, to help expand its innovative ecosystem of ideas." ${ }^{13}$ The mission of DIUx Silicon Valley is to serve as a local point to strengthen existing relationships, build new ones and scout for breakthrough and emerging technologies.

Another example of partnership is Niteworks between the MOD, the Defence Science \& Technology Laboratory (DSTL) and more than 150 UK-based companies that work together to support MOD decision-makers in the fields of operations, acquisition and capability. ${ }^{14}$ Based on the success of the partnership, the initiative has been extended to 2018. The Niteworks approach enables the MOD to rapidly assemble expertise in an impartial environment, with access to prior knowledge and industry intellectual property from across the defense community. It brings together knowledge of the problem and solution space, which both enables a better understanding of the feasibility of recommendations and allows them to be rigorously tested and challenged from a range of perspectives, blending incumbent knowledge with the fresh thinking of new suppliers - be they generated by small and medium-sized enterprises or a global company.

Similarly, in 2010, 2012 and 2014, the Brussels-based think tank, Friends of Europe (formerly Security and Defence Agenda), conducted Security Jams to discuss global security. ${ }^{15}$ This included brainstorming a broad range of security issues from security in Afghanistan to countering piracy operations and collaboration with emerging security players like China and India. These security topics were discussed in online forums and brought together a diverse set of thousands of security professionals from around the world. Each Security Jam resulted in a list of top ten recommendations for the NATO and EU leadership. The Security Jams are unique in the sense that they reach far beyond the usual suspects and discuss security matters in an unclassified, open environment.

\section{Challenges to Effective Public-Private Collaboration on Innovation}

Numerous articles have been written about the issues and challenges of driving innovation through partnerships between government and industry. ${ }^{16}$ The key obstacles from an industry perspective seem to involve the application of acquisition rules, a mismatch in corporate cultures and lagging timelines. The biggest issue with the acquisition rules is that the technology cycles far outpace

13 diux.mil.

14 niteworks.net.

15 friendsofeurope.org.

16 See, for example, http://www.govtech.com/local/4-Key-Challenges-Facing-LocalGovernment-Innovators.html 
the acquisition timelines. Too often, industry must work with outdated requirements during the tendering process. While acquisition rules intend to safeguard a level playing field and equal opportunities for bidders, they also take away some of the motivation to get involved in the pre-competitive phase of the procurement, during which innovations and new technologies can be discussed; there is an incentive to wait for the acquisition to be published and avoid the costs involved in the collaborative phase before the tender.

However, it is important not to confuse activity with results, as these have different meanings for different stakeholders. Businesses express results in terms of innovation, revenues, profits and growth. Governments have a different set of metrics to judge output and results. This also means that risks are assessed differently by business leaders and governments. Government leaders should offer incentives for trying new approaches and, even better, for succeeding. Punishing failure will inhibit innovation. These differences in culture make public-private sector collaboration more difficult.

A mismatch in timelines and sense of urgency hampers collaboration as well, and especially precludes the involvement of small and medium-sized enterprises. The private sector operates against the cadence of the (financial) markets with a strong focus on the bottom-line. These issues are not easy to overcome, but a number of steps can be taken to improve the overall climate in which partnering and collaboration occurs. The first is to take a programmatic approach to partnering and collaboration. Governments interact with industry through multiple groups and stakeholders, which requires some level of coordination. It is simply too expensive for industry to keep collaborating in a haphazard way, especially if the timelines are long. The second step would be to communicate openly and often and create a feedback loop. It is crucial for industry to understand what has been done with the information which is received from the industrial partners; this is part of the incentive model. Feedback regarding what the government likes about certain recommendations is important, just as is the feedback what the government does not like. A third step would involve ceasing activities that do not lead to results. Industry faces less of a problem stopping operations than if it continues with activities that produce no results. A fourth helpful step would be to establish a governance mechanism with industry to discuss collaborative activities. This would involve treating the industrial sector as a real partner and bringing small and mediumenterprises on board.

\section{Recommendations and Policy Considerations to Overcome Innova- tion Challenges}

While most government organizations are not concerned with market share or fending off competitors, they are focused on delivering services and operating in complex and dynamic environments where demand and the expectations of their constituents are increasing rapidly. As such, it is imperative for government organizations to create panoramic perspectives to better understand the 
complex operating environments (both physical and digital) in which they operate and to better understand the needs of their constituents.

The 2015 CEO Study ${ }^{17}$ provides a clear set of recommendations about dealing with technologies and innovation:

\section{Form your own futures squad}

Set up a specialist forecasting team, equipped with the right technologies and skills. Recent research shows people trained to use probabilistic reasoning techniques, and recognize and eliminate bias, produce better forecasts. Working in teams likewise increases the odds of predicting the future accurately. Consider designating someone within your organization or agency specifically to scan for new technologies and monitor the marketplace.

Set up an innovation center outside the current organizational structure for incubating and piloting new business models and offerings. Give it the latitude to experiment properly, including sufficient time and resources. Test the most promising prototypes on a select group of knowledgeable, impassioned customers and constituents. And be ruthless about discarding all but the very best options.

\section{Cultivate your cognitive capabilities}

There's no technology that can tell you exactly what will happen in the future. However, using predictive and cognitive analytics to scrutinize the real-time data you receive from across your extended enterprise and mission area will help you forecast what might happen with a greater level of confidence. It will also enable you to generate "what-if" scenarios and risk assessments, allowing you to prepare for different outcomes before they occur.

\section{Take an ecocentric view of the world}

Concentrate on building broader networks and look at what organizations in unrelated industries are doing to get completely different ideas. Assess the caliber of all the organizations and enterprises in your ecosystem. Are you leveraging all their contacts, skills and assets? Are there any weak links? Are there any missing skills? Ask yourself whether your ecosystem has the right expertise to exploit new trends and technologies and boost its power to compete. If not, where should you look? The fate of your organization now rests on the collective abilities of the ecosystem in which you operate, including its ability to read-and prepare for-the future.

All recommendations depend on two essential enablers to deal with disruptive innovation in government organizations: an innovation ecosystem and an innovation platform. Without both, innovation will to fail.

The ecosystem or community of innovators from government agencies, non-profits and the private sector should come together and rally behind a common shared perspective of the operating environment. This community

17 IBM Institute for Business Value, Redefining Boundaries, 29. 
will not sustain itself unless it is supported by a platform and venue (physical and virtual) for innovation and problem-solving. An innovation platform provides the structure for knowledge exchange and facilitates the problem-solving process. Given the rate of unprecedented technological change, governments, militaries and businesses must find creative ways to work and innovate together as described above. The trick is to overcome the dominant logic or thinking in the defense and security industry and explore disruptive innovations.

\section{About the author}

Leendert van Bochoven is member of the global leadership team for IBM's Public Sector. He is the global lead for National Security, focused on serving clients in Defence, Intelligence and Public Safety. He is also leading IBM's engagement with NATO and European Defence Agency.

In June 2015, Leendert became member of IBM's Industry Academy. The Industry Academy was established during IBM's centennial year (2011). The goal is to help increase IBM's industry capabilities internally, advance IBM's industry thought leadership and brand recognition externally, and connect transformative insights across industries to deliver client transformation.

Leendert is member of the Board of Directors of AFCEA and member of the American Defense Industries Forum in Brussels. He has a degree in Business Economics from the Erasmus University in Rotterdam, the Netherlands.

E-mail: L_van_Bochoven@nl.ibm.com 